\title{
The unsolved case of "bone-impairing analgesics": the endocrine effects of opioids on bone metabolism
}

This article was published in the following Dove Press journal:

Therapeutics and Clinical Risk Management

3I March 2015

Number of times this article has been viewed

\section{Flaminia Coluzzi ${ }^{1,2}$ \\ Joseph Pergolizzi ${ }^{3,4}$ \\ Robert B Raffa ${ }^{5}$ \\ Consalvo Mattia ${ }^{1,2}$}

'Department of Medical and Surgical Sciences and Biotechnologies, Unit of Anesthesiology, Intensive Care Medicine and Pain Therapy, Faculty of Pharmacy and Medicine - Polo Pontino, Sapienza University of Rome, Latina, Italy; ${ }^{2}$ SIAARTI Study Group on Acute and Chronic Pain, Rome, Italy; ${ }^{3}$ Department of Medicine, Johns Hopkins University School of Medicine, Baltimore, MD, ${ }^{4}$ Naples Anesthesia and Pain Associates, Naples, FL, ${ }^{5}$ Department of Pharmaceutical Sciences, Temple University School of Pharmacy, Philadelphia, PA, USA
Correspondence: Flaminia Coluzzi Department of Medical and Surgical Sciences and Biotechnologies, Unit of Anesthesiology, Intensive Care Medicine and Pain Therapy, Faculty of Pharmacy and Medicine - Polo Pontino, Sapienza University of Rome, Corso Della Repubblica 79, 04I00 Latina, Italy Tel +390773 65I 3334

Fax +390773 65I 3333

Email flaminia.coluzzi@uniroma l.it

\begin{abstract}
The current literature describes the possible risks for bone fracture in chronic analgesics users. There are three main hypotheses that could explain the increased risk of fracture associated with central analgesics, such as opioids: 1) the increased risk of falls caused by central nervous system effects, including sedation and dizziness; 2) reduced bone mass density caused by the direct opioid effect on osteoblasts; and 3) chronic opioid-induced hypogonadism. The impact of opioids varies by sex and among the type of opioid used (less, for example, for tapentadol and buprenorphine). Opioid-associated androgen deficiency is correlated with an increased risk of osteoporosis; thus, despite that standards have not been established for monitoring and treating opioid-induced hypogonadism or hypoadrenalism, all patients chronically taking opioids (particularly at doses $\geq 100 \mathrm{mg}$ morphine daily) should be monitored for the early detection of hormonal impairment and low bone mass density.
\end{abstract}

Keywords: opioids side effects, bone metabolism, fractures, OPIAD, endocrine system, chronic pain

\section{Introduction}

Severe chronic pain still represents a challenge for physicians, as it profoundly affects not only the quality of life of patients and their health and mental status, but it also impacts their ability to work and represents a huge economic burden for our society. Data from a European survey suggest that about $25 \%$ of the population experiences chronic pain that affects the muscles, joints, neck, or back, lasting for more than 3 months. ${ }^{1}$ The increased prevalence of other comorbidities, such as anxiety and depression, may further exacerbate the impact of chronic pain on patients and may decrease their physical and mental functioning. ${ }^{2}$ Chronic pain has been recognized as involving the phenomena of central sensitization and neuroplasticity, which alter the physiological transduction and perception of nociceptive stimuli. ${ }^{3}$

In clinical practice, pain is classified as acute or chronic, only on the basis of its duration, and analgesics are often chosen only on the basis of pain intensity (eg, mild, moderate, or severe), as suggested by the World Health Organization's analgesic ladder. ${ }^{4}$ This approach is, however, not informative, because pain intensity does not reflect the neurobiological cause. Chronic pain is an expression of a dysfunction of the peripheral or central nervous system (CNS); therefore, treatment strategies should shift from the existing strategy of symptom control to a mechanism-oriented approach. This new approach represents an epochal change in the management of chronic pain ${ }^{5,6}$ and opens the door to a wider use of central analgesics, including opioids, which better fit with the real needs of chronic pain patients. 
Unfortunately, the recent literature suggests the possible risks to bone and its functions in chronic opioid analgesics users. ${ }^{7}$ This review aims to analyze the current hypotheses by which opioids could be considered "bone-impairing analgesics".

\section{The first possible connection: increased number of falls}

In recent years significant differences have been observed between subjects exposed to long-term pain medication use and nonusers in terms of the incidence of bone fractures and fall-related injuries. This supports the idea that analgesics, particularly centrally acting drugs, may indirectly affect bone through fall-related fractures caused by effects on the CNS, such as dizziness and sedation. Among analgesics, opioids have been frequently associated with an increased risk of fall-related fractures, particularly in the elderly. ${ }^{8}$ Indeed, fall-related fractures are the sixth leading cause of death among older adults. ${ }^{9}$

Managing chronic pain in the elderly by using weak opioids or low doses of strong opioids may be safer than using nonsteroidal anti-inflammatory drugs (NSAIDs) because opioids do not impair organ function, which may be declined in these patients. ${ }^{10}$ However, opioids may increase somnolence and confusion, which play a role in increasing the risk of falls of people in older age. ${ }^{11}$ For example, Ensrud et $\mathrm{al}^{12}$ evaluated a cohort of more than 8,000 elderly women taking narcotics and reported a significant increase in the risk of nonspine fractures (hazard ratio [HR]: 1.40), probably related to the cognitive effects of such drugs. Similar results have been found in Denmark in a nationwide registry-based study of about 125,000 cases of fractures: all examined opioids increased the risk of fractures, except buprenorphine, pethidine, dextropropoxyphene, and combinations of acetylsalicylic acid and codeine. ${ }^{13}$ The increased incidence of fractures was observed, even in the first days of treatment, during the titration phase, when opioid doses are low. Therefore, despite most of these fractures involving typical osteoporotic localizations such as the spine, hip, and forearm, it is unlikely that they could be the result of a direct effect on bone structure - they are more likely related to the risk of falls due to CNS effects. ${ }^{13}$ A recent epidemiological study based on the United Kingdom General Practice Research Database, where data between January 1, 1990 and December 31, 2008 have been analyzed, showed a direct relationship between the cumulative dose of opioid administration and risk of fracture. However, while a single opioid prescription increased the risk about threefold (odds ratio [OR]: 2.70; 95\% confidence interval [CI]: 2.34-3.13), the risk decreased (OR: 1.17; 95\% CI: 1.08-1.27) when opioid therapy was maintained over time. Patients taking more than 20 prescriptions of opioids or patients that ended opioid use more than 1 month before injuries had no increased risk of fracture. These results support the hypothesis of the CNS effects of opioids, which most frequently occur during titration, when the right dose for each patient has to be found. Usually, CNS symptoms resolve in the first days/weeks of treatment because of adequate titration or even opioid tolerance development; however, special precautions should be taken in frail individuals, such as the elderly, patients with dementia, or people taking other sedating medications. ${ }^{14}$ According to a Canadian study of 400,000 patients, there is a wide use of drugs with sedative effects among the elderly (at least one drug prescribed in more than $50 \%$ of the study population over a 1 -year follow-up). ${ }^{8}$ Among the examined cases, $15.3 \%$ were taking opioids and $20.7 \%$ were taking more than one sedative medication. The incidence of injuries was relatively low $(3.7 \%)$, but the majority were fractures (55.1\%). When considering second-step opioids, combinations containing codeine were associated with the highest risk of injury (HR: 2.27). ${ }^{8}$ These studies provide evidence of a relationship between opioid and fall-related injury. This is probably related to an effect on the CNS, such as dizziness or an altered state of consciousness.

Therefore, opioids are not the only CNS drugs to be associated with an increased risk of fractures and fallrelated injuries; antipsychotic agents, benzodiazepines, ${ }^{15}$ and antidepressants may also increase the risk of fractures by 25\% (HR: 1.25). ${ }^{12}$ However, both the likelihood of weakened bones and multiple medications in the elderly increase the risk of fractures.

A further confounding factor comes from the evidence that NSAID users also have a slightly increased risk of fractures when compared to nonusers (HR: 1.44). In a 10-year follow-up of more than 2,000 perimenopausal women taking analgesics, no statistically significant differences were observed in bone mineral density (BMD) alterations, but NSAID users were more prone to fractures, while the use of paracetamol and opioids was not associated with a significant trend toward more fractures. Similarly, no increased risk was recorded for aspirin users. ${ }^{16}$

\section{The second possible connection: reduced bone density}

A more recent approach to understanding the increased risk of fractures among chronic analgesics users includes the evaluation of the quality of the broken bone and, therefore, 
the close relationship between pain medication use, endocrine changes, and reduced bone density.

Bone is a living tissue that undergoes a remodeling process in order to maintain a balance between formation and resorption. Two types of cells are involved in this process: the mesenchymal-derived osteoblasts, which synthesize new bone matrix; and the monocyte/macrophage-derived osteoclasts (for bone resorption). Osteoclasts express cathepsin K, vitronectin receptor, and tartrate-resistant acid phosphatase, whereas osteoblasts express alkaline phosphatase, type I collagen, and osteocalcin. Osteocytes are cells of the mature bone, derived from osteoblasts, embedded within the mineralized bone matrix. The activity of these cells is regulated by numerous factors, including cytokines in the local environment, vitamins, growth factors, and systemic hormones. ${ }^{17}$

Bone remodeling, which is essential to maintain bone strength, consists of four phases: activation; resorption; reversal; and formation. On the surface of trabecular bone or in cortical bone, osteoclasts are activated and initiate the "resorption" phase, during which there are only mononuclear cells. The "reversal" phase is an intermediate period between osteoclast-mediated resorption and osteoblast-mediated formation, during which osteogenic signals released by osteoclasts activate the bone surface and bone marrow cells; they also guarantee the coupling process. Once osteoblasts are recruited, the "formation" phase starts, during which osteoblasts produce bone matrix. When the precise balance of these four phases is lost in favor of resorption, the result is bone loss, as in a number of bone diseases, including osteoporosis. ${ }^{18,19}$ In addition to bisphosphonates, which represent the current gold standard of therapy, a number of potential new drugs are under investigation for the treatment of osteoporosis and bone metastases. New molecular targets have been identified in bone biology, such as the cytokine receptor triad formed by the receptor activator of nuclear factor kappa-B (NF-kB) ligand (RANKL)-receptor activator of NF-kB (RANK)osteoprotegerin, for which a drug is already available for clinical use (denosumab), and others for which medications are still in Phase I, II, or III of development. ${ }^{20-22}$

Medication-induced osteoporosis is an emerging health problem related to a number of commonly used drugs, such as selective serotonin receptor inhibitors, anticonvulsants, glucocorticoids, proton pump inhibitors, and others. Chronic use of these medications increases the risk of reduced bone mineral density (BMD) and fractures, particularly when patients are polymedicated with more than one of these drugs. ${ }^{23}$

Opioids have been recognized as a risk factor for the development of osteoporosis for more than 10 years. ${ }^{24}$ Other
CNS-active medications, such as anticonvulsants, have also been associated with a significant reduction in BMD, while benzodiazepines and antidepressants have not. ${ }^{25}$ On antidepressants in particular, the evidence is discordant, as a recent study on male osteoporosis showed that opioids, glucocorticoids, and also antidepressants increased osteoporosis risk. $^{26}$

The mechanism by which opioids increase the risk of fractures by interfering with bone density is related to both direct and indirect effects on bone metabolism: 1) by the direct presence of opioid receptors on osteoblasts; and 2) by the interference with the complex mechanisms that physiologically regulate bone turnover (Figure 1).

A number of experimental studies on animal and human cells show that opioids can reduce BMD by directly interfering with osteoblast activity. ${ }^{27-29}$ Indeed, opioid receptors that are present on murine osteoblasts and proenkephalin-derived peptides may inhibit the activity of alkaline phosphatase in rat osteosarcoma-derived cell lines. ${ }^{28}$ Similarly, in humans, opioids are thought to interfere with the growth of osteoblasts since they reduced the levels of serum osteocalcin, which is a marker of osteoblast activity. In human osteoblast-like cells (MG-63), all the three types of opioid receptor subtypes have been identified: mu (MOR); delta (DOR); and kappa (KOR). When incubated with morphine, osteocalcin synthesis is significantly reduced, while alkaline phosphatase secretion is not affected, and naloxone completely reverses the effect of morphine on these cells. ${ }^{27}$ Similarly, there are low serum osteocalcin levels in heroin- and cocaine-abusing women when pregnant, as well as in the umbilical arteries of their newborns, suggesting a toxic effect of these illicit drugs on osteoblasts. ${ }^{29}$

There seem to be some exceptions, or at least reduced effects, among analgesics. For example, in adult female rats, chronic use of tramadol was shown to have a lower osteoporotic effect than morphine or fentanyl. ${ }^{30}$ This might be explained by the lower affinity for MOR compared to the other agonists. It might also relate to the multimechanistic nature of tramadol (opioid plus nonopioid). If so, the osteoporotic potential of different opioids may vary, and the choice of drug can be crucial, particularly in patients with a wellknown diagnosis of, or at an increased risk of, osteoporosis. ${ }^{30}$ Accordingly, it is reasonable to think that analgesics with dual mechanisms of action, such as tapentadol or even buprenorphine, could have a better profile in reducing the impact of opioids on bone density. However, further clinical studies are warranted to support these findings.

Evidence also indicates that opioids influence the complex system that physiologically modulates bone turnover, 


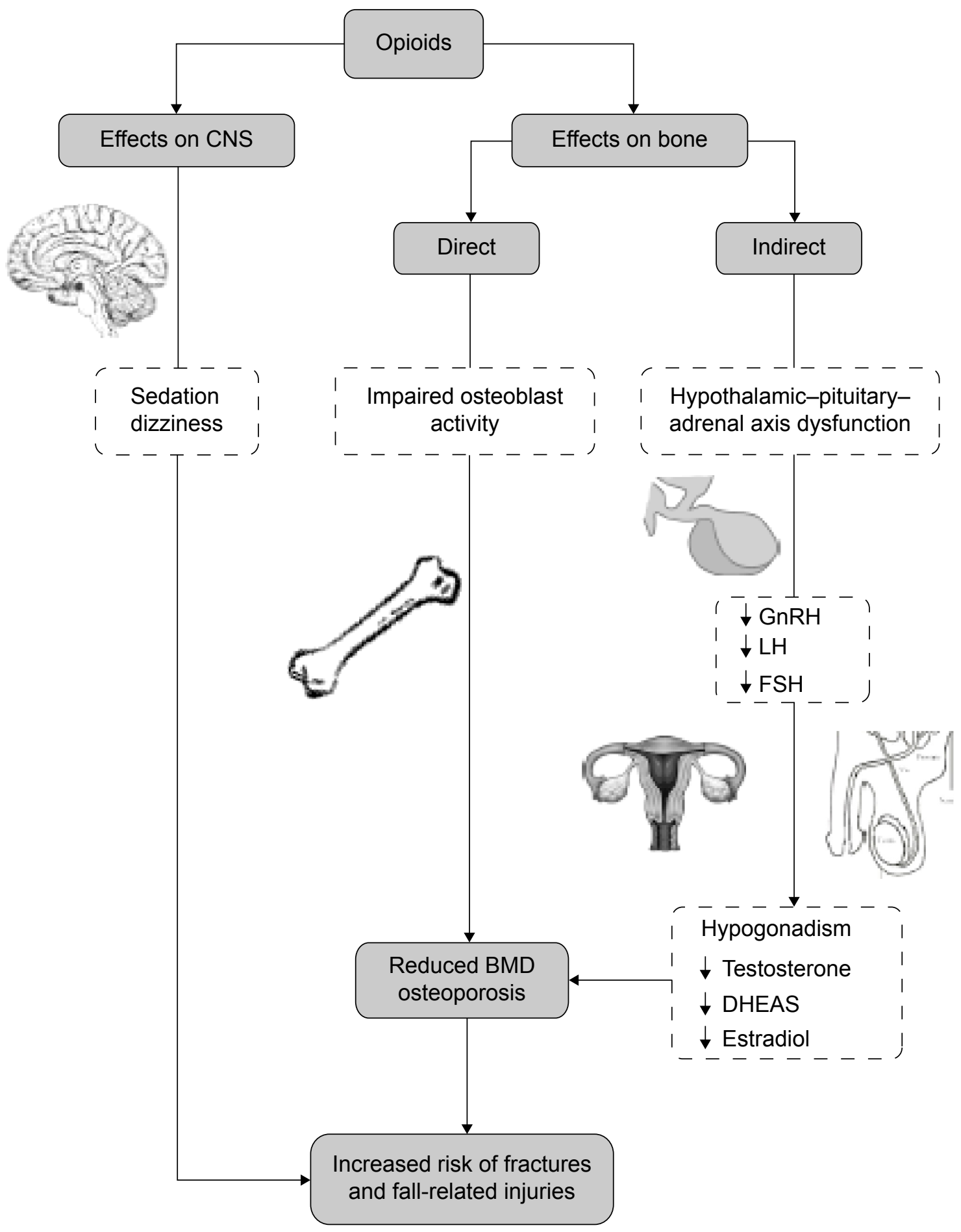

Figure I Mechanisms by which opioids may increase the risk of fracture and fall-related injuries.

Abbreviations: CNS, central nervous system; GnRH, gonadotropin-releasing hormone; LH, luteinizing hormone; FSH, follicle-stimulating hormone; DHEAS, dehydroepiandrosterone sulfate; BMD, bone mass density.

leading to opioid-induced osteoporosis and opioid-associated bone fractures. Most data come from patients using intrathecal opioids and from heroin addicts; these subjects are exposed to an increased risk of endocrinopathies, which are often undetected. Usually, these effects are reversible, but chronic use may lead to serious symptoms when the diagnosis is missed. ${ }^{31,32}$

The endocrine effects of opioids primarily include the inhibition of the hypothalamic, pituitary, and gonadal hormones, and hypothalamic-pituitary-adrenal axis 
dysfunction. ${ }^{33}$ Both sexes are exposed to this risk; hypogonadism has been observed after the chronic use of opioids, and it is related to the central suppression of the hypothalamic secretion of gonadotropin-releasing hormone; decreased pituitary luteinizing hormone (LH), follicle-stimulating hormone (FSH), adrenal dehydroepiandrosterone and testosterone, and progesterone and estradiol in women; as well as decreased testicular testosterone in men. ${ }^{34}$

In women, ovarian sex hormones may be inhibited by the chronic use of opioids; in premenopausal patients, LH and FSH may be reduced by $30 \%$, while in postmenopausal patients, they may be reduced to only one-third of normal. Similarly, adrenal androgen production, measured as the free testosterone level, may be reduced by $40 \%$ in opioid users. ${ }^{35}$ This opioid-induced "hormone dysregulation", observed with controlled-release oral and transdermal formulations, may lead to menstrual irregularities, reduced fertility, sexual dysfunction, fatigue, depression, a loss of muscle strength and mass, and osteoporosis.

In menopause, there is a natural decline in estrogen levels, which increases the risk of osteoporosis, characterized by low BMD and deterioration of the architectural structure of the bones. ${ }^{18}$ This is the main reason why up to $50 \%$ of women are likely to have a fracture after the age of 50 years, compared to only $20 \%$ of men. ${ }^{36}$ Indeed, the incidence of osteoporosis is significantly lower in men; they are protected against osteoporotic fractures by greater bone strength, in terms of both cortical bone expansion and trabecular bone volume, which matured during the pubertal time and have been maintained during aging by sufficient estrogen levels. Androgens exert further protection against fractures via their anabolic effects on muscle mass. ${ }^{36}$

Chronic opioid users are prone to develop alterations in the hypothalamic-pituitary-gonadal axis, which can result in hypogonadotropic hypogonadism. This condition, named opioid-induced androgen deficiency (OPIAD), has been observed in more than 5 million men suffering from chronic noncancer pain in the Unites States. ${ }^{37}$ These patients experience decreased libido, erectile dysfunction, depression, fatigue, hot flashes, and reduced quality of life. Untreated patients with OPIAD may develop further complications such as osteopenia or osteoporosis. ${ }^{37}$ A number of androgen replacement therapy options have been developed; however, unfortunately, OPIAD is often unrecognized and underdiagnosed. In men with OPIAD, testosterone patch therapy at a dose of $7.5 \mathrm{mg} /$ day has been reported to significantly improve androgen deficiency symptoms, sexual function, depression score, and quality of life. ${ }^{38}$ Testosterone or estradiol levels were found to be below the normal range in men chronically taking sustained-action oral opioids for chronic noncancer pain. Androgen levels approached the castrate range, with consequent severe erectile dysfunction in about $90 \%$ of the patients. These patients experienced weakness and reduced muscular mass, which can increase the risk of bone fracture. Moreover, they had reduced levels of dehydroepiandrosterone sulfate (DHEAS), a marker of generalized adrenal insufficiency; two-thirds of cases had DHEAS values below age-specific norms, and one-third had values below $15 \mu \mathrm{g} / \mathrm{dL}$, which is the detection limit of laboratory tests. DHEAS deficiency was not accompanied by adrenocorticotropic hormone (ACTH) deficiency, supporting the hypothesis of opioid-induced inhibition of adrenal androgen production, rather than reduced ACTH stimulation. ${ }^{39,40}$ Experimental studies have shown that the gonads are directly affected by morphine treatment, which modify the expression of testicular and ovarian estrogen and androgen receptors in rats. ${ }^{41}$

Besides hypogonadotropic hypogonadism or decreased adrenal androgen production, sustained-release opioids also induce cortisol deficiency, identified by decreased levels of cortisol and decreased cortisol response to corticotropin. ${ }^{42}$ Opioids reduce the concentration of corticotropin-releasing hormone and the secretion of ACTH from the pituitary. However, while sex hormone deficiency causes well-known symptoms (known as OPIAD), the clinical significance of opioid-induced cortisol deficiency is still unclear and may range from fatigue and depression to clinical features that mimic adrenocortical failure. ${ }^{43}$ The interaction between glucocorticoids and opioids in nociception is currently an objective of scientific research. Evidence suggests a clear overlapping between the two analgesic systems: opioids regulate glucocorticoid-induced analgesia and glucocorticoids regulate opioid-induced analgesia. ${ }^{44}$

It is still unclear whether or not opioid-induced hypogonadism is associated with an increased risk of bone fractures. ${ }^{45}$ Few available data come from studies on addicts receiving methadone maintenance treatment (MMT). The first report by Pedrazzoni et $\mathrm{al}^{46}$ showed that among male chronic heroin users, the more recent the last dose, the more likely a low BMD. Abusers, who had their last doses less than 2 days before the evaluation showed a significantly increased risk of lower vertebral BMD $(P<0.05)$ compared with former addicts who were drug-free for 4-24 months prior. ${ }^{46}$ Similarly, in a cohort of participants in an MMT program, $83 \%$ of enrolled patients had a low BMD, with a $T$-score $<-2.5$, as measured by dual-energy X-ray densitometry, and male sex was a significant predictor of low BMD. ${ }^{47}$ 
Recently, BMD and markers of bone turnovers were evaluated in 83 patients receiving MMT for a median of 11 years. BMD was lower than normal (approximately 10\% below the population mean) only in men, but not in women, while markers of bone turnover were normal in both sexes. Men taking MMT had a lower serum testosterone level compared with controls, with possible increases in the risk of fracture. ${ }^{48}$ In aging patients with HIV, heroin use and MMT were associated with lower BMD in the lumbar spine in both sexes. ${ }^{49,50}$ According to these findings, the chronic use of opioids (methadone) significantly increases the risk of low BMD; however, the reported differences among the sexes are still difficult to explain. Which factors could contribute to the observed increased risk in men? Tobacco smoking, a recognized risk factor for osteoporosis and a very common comorbidity among MMT participants, is unlikely to explain the observed differences between sexes, because the incidence of active smokers was similar between men and women in the reported studies. Perhaps estrogens could be critical in men, as in females, for bone homeostasis. ${ }^{51}$ Differences in gonadal function need further investigation in order to explain the effects of opioids on bone metabolism.

Hypogonadism is a well-recognized factor for lower BMD because sexual hormones influence bone mass and integrity, and protect against osteoporosis. ${ }^{52}$ Low serum testosterone levels have been reported in men taking heroin and high-dose MMT, presumably due to the opioid suppression of gonadotropin secretion. This effect is reversed by naloxone. ${ }^{53}$ Among long-term oral opioid users, the prevalence of osteopenia and hypogonadism was significantly higher in men than in women $-50 \%$ of men versus $21 \%$ of premenopausal women had a $T$-score between -1 and -2.5 , whereas the prevalence of hypogonadism was more than threefold in men than in women ( $75 \%$ versus $21 \%$, respectively). ${ }^{54}$ These results showed a higher percentage of hypogonadism (75\%) compared with osteopenia $(50 \%)$ in male subjects, suggesting that other factors should contribute to the effects of opioids on bone quality. However, other studies showed exactly the opposite result; in a cohort of 81 male patients using opioids, about half of the patients had osteopenia/ osteoporosis, but only $27 \%$ were hypogonadal. ${ }^{55}$ Therefore, testosterone levels are not definitive for an increased risk of low BMD and related fractures. Additional factors should be considered.

To summarize the aforementioned findings, hypogonadism is likely the main cause of osteoporosis in both sexes. However, the current knowledge is still inadequate to clarify why men are more likely to be affected during the chronic use of opioids than women, and by which mechanisms opiates exert this effect. Opioid-induced hypogonadism is underrecognized and undertreated. So, a major lesson we can learn from the data is the need for monitoring hormone levels, ${ }^{56}$ and for routinely screening $\mathrm{BMD}^{57}$ in all patients during chronic opioid therapy. Standards have not been established for monitoring and treating opioid-induced hypogonadism or hypoadrenalism. Based on the literature and clinical experience, patients taking opioid therapy equivalent to $\geq 100 \mathrm{mg}$ of daily morphine should be monitored for the development of hypogonadism. Suggested laboratory tests that can be useful to check endocrine system function include: total and free testosterone levels; and blood concentrations of sex hormone-binding globulin, LH, FSH, DHEAS, and estradiol (in women). ${ }^{42,57}$

\section{Not all opioids produce equal effects on the hypothalamic- pituitary-gonadal axis}

In view of the well-known suppressive effects, clinicians should evaluate the different abilities of individual opioids on their ability to affect the hypothalamic-pituitary-gonadal axis (and in individual patients). Preclinical studies on male rats found no difference among the opioids morphine, fentanyl, tramadol, and buprenorphine on plasma testosterone levels at 4 hours after a single injection; however, testosterone was significantly reduced when animals were treated with high concentrations of the opioids tested. ${ }^{58}$ Brain testosterone levels were significantly reduced when compared to control (saline) 4 hours after morphine, fentanyl, or tramadol injection, but not after buprenorphine (Figure 2). It has been speculated that the different effect of buprenorphine might be due to the relatively slow initial binding to MOR and longer receptor occupancy when compared with other opioids. ${ }^{58}$

Similar findings have been reported in clinical studies on patients treated with intrathecal morphine and with the long-term use of buprenorphine. Epidural morphine was shown to decrease testosterone plasma levels in both sexes, which was completely reversed after the interruption of therapy, and this was related to a peripheral action (on the testis in men and in the adrenals in men and women). In contrast, when patients were treated with buprenorphine for 6 months, only free testosterone was reported to be lower than baseline in men - an effect paralleled by the increase of sex hormone binding globuline - while no effects were recorded in any of the hormones in women. ${ }^{59}$ In women of different reproductive ages, the hormonal effects of transdermal buprenorphine have been reported to be negligible in clinical 

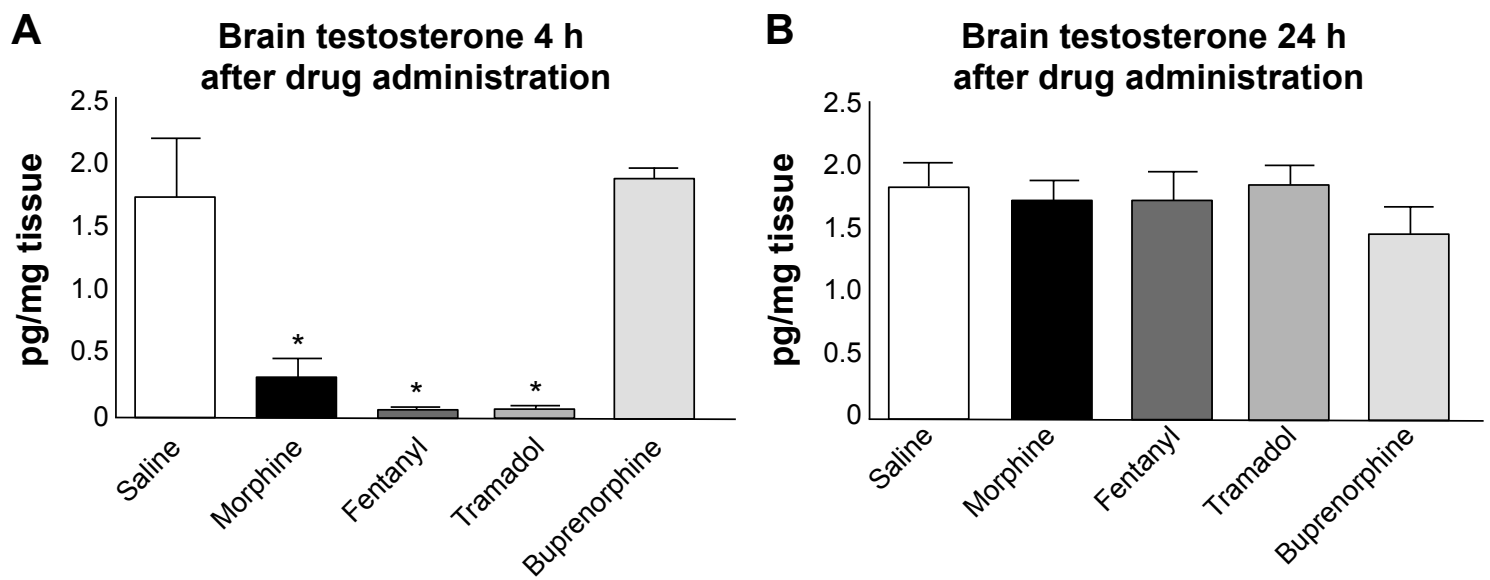

Figure 2 Opioid-induced effects on brain testosterone.

Notes: *Diencephalic brain testosterone levels following the subcutaneous administration of morphine ( $10 \mathrm{mg} / \mathrm{kg})$, fentanyl $(0.1 \mathrm{mg} / \mathrm{kg})$, tramadol (40 mg/kg), or buprenorphine $(0.1 \mathrm{mg} / \mathrm{kg})$. (A) Brain testosterone levels were lower than control in all groups except buprenorphine at 4 hours. (B) No differences were observed between groups in brain testosterone levels at 24 hours. Reprinted from Neuroscience, I40(3), Ceccarelli I, De Padova AM, Fiorenzani P, Massafra C, Aloisi AM; Single opioid administration modifies gonadal steroids in both the CNS and plasma of male rats, 929-937, Copyright (2006), with permission from Elsevier. ${ }^{58}$

Abbreviation: h, hours.

practice, with no side effects suggestive of hypogonadism. Total and free testosterone were not reduced during standard treatment with the buprenorphine transdermal system, $35 \mu \mathrm{g} /$ hour administered every 72 hours. ${ }^{60}$

Tapentadol is an innovative opioid with two mechanisms of action: MOR agonism (with a 50-fold lower affinity for the human MOR than morphine); and norepinephrine reuptake inhibition (the first of a new pharmacological class named MOR/noradrenaline reuptake inhibitor). The results from three randomized placebo-controlled studies involving healthy subjects or patients with osteoarthritis knee pain showed that the effects of tapentadol treatment on testosterone and gonadotropin ( $\mathrm{LH}$ and/or FSH) levels were minimal and not considered to be clinically relevant. The reason for these findings is not completely clear; however, it is likely that a difference in the effects of tapentadol on sexual hormones when compared with morphine may be related to the dual mechanism of action of tapentadol (opioid and nonopioid) ${ }^{61}$ The contribution of the nonopioid component to analgesia results in a mechanistic "opioid-sparing" effect and the nonopioid component might mitigate against some opioid-induced adverse effects. Sufficient clinical or preclinical data on this point are lacking; however, it is clear that the lower the MOR activity, the lower the negative impact on the hypothalamic-pituitary-gonadal axis.

\section{Conclusion}

Chronic pain can be managed by using analgesic drugs and nonpharmacological techniques. Opioids are currently the cornerstone in the pharmacologic treatment of severe chronic pain. However, in addition to the desired analgesic effect, they lead to a number of side effects, such as nausea, vomiting, constipation, respiratory depression, sedation, and cognitive impairment. The onset of intolerable side effects often leads to the decision to reduce drug dosage, raising the risk of inadequate analgesia, leading to the establishment of a dangerous "vicious cycle". ${ }^{5}$ However, besides the wellknown undesirable adverse effects, there are other clinically less visible and still poorly understood nonanalgesic actions, such as their effects on bone metabolism. These are less studied and are underdiagnosed. ${ }^{6}$ The risk of misunderstanding these relevant aspects of chronic opioid treatment may lead to a number of comorbidities, such as hypogonadism and osteoporosis. Different studies have shown that both men and women chronically treated with opioids are at an increased risk for a reduction in BMD. Preclinical studies have shown that some opioids have a lower osteoporotic effect than others; however, it is still unclear which opioids are less likely to cause endocrine dysfunction and posses the best profile for minimizing the negative effects on bone metabolism. Similarly, more studies are needed to identify which patients need to be screened, what length of time is needed to develop endocrine dysfunction after starting opioids, and if these effects are reversible after stopping opioid therapy. It is reasonable to think that analgesics that have a lower contribution of MOR activity to the overall analgesic effect could represent the best choice in the delicate balance between analgesic efficacy and adverse effects. If further studies confirm this hypothesis, the choice of analgesic drug for chronic pain management should also take into consideration this particular endocrinological-metabolic aspect, especially in predisposed, frail, and elderly patients. 


\section{Disclosure}

Flaminia Coluzzi and Consalvo Mattia serve as consultants, speakers, and members of advisory boards for several pharmaceutical companies. Joseph Pergolizzi and Robert B Raffa are speakers, consultants, and/or basic science investigators for several pharmaceutical companies involved in analgesic research, but they receive no royalties (cash or otherwise) from the sale of any products. The authors report no other conflicts of interest in this work.

\section{References}

1. Breivik H, Collett B, Ventafridda V, Cohen R, Gallacher D. Survey of chronic pain in Europe: prevalence, impact on daily life, and treatment. Eur J Pain. 2006;10(4):287-333.

2. Langley PC. The prevalence, correlates and treatment of pain in the European Union. Curr Med Res Opin. 2011;27(2):463-480.

3. Woolf CJ; American College of Physicians; American Physiological Society. Pain: moving from symptom control toward mechanismspecific pharmacological management. Ann Intern Med. 2004;140(6): $441-451$.

4. Ventafridda V, Tamburini M, Caraceni A, De Conno F, Naldi F. A validation study of the WHO method for cancer pain relief. Cancer. 1987; 59(4):850-856.

5. Coluzzi F, Berti M. Change Pain: changing the approach to chronic pain. Minerva Med. 2011;102(4):289-307.

6. Pergolizzi J, Ahlbeck K, Aldington D, et al. The chronic pain conundrum: should we CHANGE from relying on past history to assessing prognostic factors? Curr Med Res Opin. 2012;28(2):249-256.

7. Mattia C, Di Bussolo E, Coluzzi F. Non-analgesic effects of opioids: the interaction of opioids with bone and joints. Curr Pharm Des. 2012; 18(37):6005-6009.

8. Buckeridge D, Huang A, Hanley J, et al. Risk of injury associated with opioid use in older adults. J Am Geriatr Soc. 2010;58(9):1664-1670.

9. Rubenstein LZ, Josephson KR. Falls and their prevention in elderly people: what does the evidence show? Med Clin North Am. 2006;90(5): $807-824$.

10. Kress HG, Ahlbeck K, Aldington D, et al. Managing chronic pain in elderly patients requires a CHANGE of approach. Curr Med Res Opin. 2014;30(6):1153-1164.

11. Dziechciaż M, Balicka-Adamik L, Filip R. The problem of pain in old age. Ann Agric Environ Med. 2013;Spec no. 1:35-38.

12. Ensrud KE, Blackwell T, Mangione CM, et al; Study of Osteoporotic Fractures Research Group. Central nervous system active medications and risk for fractures in older women. Arch Intern Med. 2003;163(8): 949-957.

13. Vestergaard P, Rejnmark L, Mosekilde L. Fracture risk associated with the use of morphine and opiates. J Intern Med. 2006;260(1):76-87.

14. Li L, Setoguchi S, Cabral H, Jick S. Opioid use for noncancer pain and risk of fracture in adults: a nested case-control study using the general practice research database. Am J Epidemiol. 2013;178(4):559-569.

15. Landi F, Onder G, Cesari M, Barillaro C, Russo A, Bernabei R; Silver Network Home Care Study Group. Psychotropic medications and risk for falls among community-dwelling frail older people: an observational study. J Gerontol A Biol Sci Med Sci. 2005;60(5):622-626.

16. Vestergaard P, Hermann P, Jensen JE, Eiken P, Mosekilde L. Effects of paracetemol, non-steroidal anti-inflammatory drugs, acetylsalicylic acid, and opioids on bone mineral density and risk of fracture: results of the Danish Osteoporosis Prevention Study (DOPS). Osteoporos Int. 2012;23(4):1255-1265.

17. Russell RG, Espina B, Hulley P. Bone biology and the pathogenesis of osteoporosis. Curr Opin Rheumatol. 2006;18 Suppl 1:S3-S10.

18. Raisz LG. Pathogenesis of osteoporosis: concepts, conflicts, and prospects. J Clin Invest. 2005;115(12):3318-3325.
19. Delaisse JM. The reversal phase of the bone-remodeling cycle: cellular prerequisites for coupling resorption and formation. Bonekey Rep. 2014; 3:561.

20. Walsh MC, Choi Y. Biology of the RANKL-RANK-OPG system in immunity, bone, and beyond. Front Immunol. 2014;5:511.

21. Coluzzi F, Di Bussolo E, Mandatori I, Mattia C. Bone metastatic disease: taking aim at new therapeutic targets. Curr Med Chem. 2011;18(20): 3093-3115.

22. Coluzzi F, Mandatori I, Mattia C. Emerging therapies in metastatic bone pain. Expert Opin Emerg Drugs. 2011;16(3):441-458.

23. Panday K, Gona A, Humphrey MB. Medication-induced osteoporosis: screening and treatment strategies. Ther Adv Musculoskelet Dis. 2014; 6(5):185-202.

24. Daniell HW. Opioid osteoporosis. Arch Intern Med. 2004;164(3):338; author reply 338 .

25. Kinjo M, Setoguchi S, Schneeweiss S, Solomon DH. Bone mineral density in subjects using central nervous system-active medications. Am J Med. 2005;118(12):1414.

26. Nelson RE, Nebeker JR, Sauer BC, LaFleur J. Factors associated with screening or treatment initiation among male United States veterans at risk for osteoporosis fracture. Bone. 2012;50(4):983-988.

27. Pérez-Castrillón JL, Olmos JM, Gómez JJ, et al. Expression of opioid receptors in osteoblast-like MG-63 cells, and effects of different opioid agonists on alkaline phosphatase and osteocalcin secretion by these cells. Neuroendocrinology. 2000;72(3):187-194.

28. Rosen H, Bar-Shavit Z. Dual role of osteoblastic proenkephalin derived peptides in skeletal tissues. J Cell Biochem. 1994;55(3):334-339.

29. Rico H, Costales C, Cabranes JA, Escudero M. Lower serum osteocalcin levels in pregnant drug users and their newborns at the time of delivery. Obstet Gynecol. 1990;75(6):998-1000.

30. Boshra V. Evaluation of osteoporosis risk associated with chronic use of morphine, fentanyl and tramadol in adult female rats. Curr Drug Saf. 2011;6(3):159-163.

31. Elliott JA, Horton E, Fibuch EE. The endocrine effects of long-term oral opioid therapy: a case report and review of the literature. $J$ Opioid Manag. 2011;7(2):145-154.

32. Duarte RV, Raphael JH, Southall JL, Labib MH, Whallett AJ, Ashford RL. Hypogonadism and low bone mineral density in patients on long-term intrathecal opioid delivery therapy. BMJ Open. 2013:3(6).

33. Katz N, Mazer NA. The impact of opioids on the endocrine system. Clin J Pain. 2009;25(2):170-175.

34. Vuong C, Van Uum SH, O'Dell LE, Lutfy K, Friedman TC. The effects of opioids and opioid analogs on animal and human endocrine systems. Endocr Rev. 2010;31(1):98-132.

35. Daniell HW. Opioid endocrinopathy in women consuming prescribed sustained-action opioids for control of nonmalignant pain. J Pain. 2008; 9(1):28-36.

36. Vanderschueren D, Laurent MR, Claessens F, et al. Sex steroid actions in male bone. Endocr Rev. 2014;35(6):906-960.

37. Smith HS, Elliott JA. Opioid-induced androgen deficiency (OPIAD). Pain Physician. 2012;15(3 Suppl):SE145-SE156.

38. Daniell HW, Lentz R, Mazer NA. Open-label pilot study of testosterone patch therapy in men with opioid-induced androgen deficiency. J Pain. 2006;7(3):200-210.

39. Daniell HW. Hypogonadism in men consuming sustained-action oral opioids. J Pain. 2002;3(5):377-384.

40. Daniell HW. DHEAS deficiency during consumption of sustained-action prescribed opioids: evidence for opioid-induced inhibition of adrenal androgen production. J Pain. 2006;7(12):901-907.

41. Vodo S, Arcelli D, Fiorenzani P, et al. Gonadal ER $\alpha / \beta$, AR and TRPV1 gene expression: modulation by pain and morphine treatment in male and female rats. Physiol Behav. 2013;110-111:80-86.

42. Colameco S, Coren JS. Opioid-induced endocrinopathy. J Am Osteopath Assoc. 2009;109(1):20-25.

43. Policola C, Stokes V, Karavitaki N, Grossman A. Adrenal insufficiency in acute oral opiate therapy. Endocrinol Diabetes Metab Case Rep. 2014; 2014:130071. 
44. Kesmati M, Rezaie M, Fathi-Moghaddam H. Interaction between glucocorticoids and opioids in nociception in young and adult rats. Open Access Anim Physiol. 2010;2010(2):87-92.

45. Irwig MS. Bone health in hypogonadal men. Curr Opin Urol. 2014; 24(6):608-613.

46. Pedrazzoni M, Vescovi PP, Maninetti L, et al. Effects of chronic heroin abuse on bone and mineral metabolism. Acta Endocrinol (Copenh). 1993; 129(1):42-45.

47. Kim TW, Alford DP, Malabanan A, Holick MF, Samet JH. Low bone density in patients receiving methadone maintenance treatment. Drug Alcohol Depend. 2006;85(3):258-262.

48. Grey A, Rix-Trott K, Horne A, Gamble G, Bolland M, Reid IR. Decreased bone density in men on methadone maintenance therapy. Addiction. 2011;106(2):349-354.

49. Arnsten JH, Freeman R, Howard AA, Floris-Moore M, Santoro N, Schoenbaum EE. HIV infection and bone mineral density in middleaged women. Clin Infect Dis. 2006;42(7):1014-1020.

50. Arnsten JH, Freeman R, Howard AA, Floris-Moore M, Lo Y, Klein RS. Decreased bone mineral density and increased fracture risk in aging men with or at risk for HIV infection. AIDS. 2007;21(5):617-623.

51. Napoli N, Faccio R, Shrestha V, Bucchieri S, Rini GB, ArmamentoVillareal R. Estrogen metabolism modulates bone density in men. Calcif Tissue Int. 2007;80(4):227-232.

52. Vanderschueren D, Vandenput L, Boonen S, Lindberg MK, Bouillon R, Ohlsson C. Androgens and bone. Endocr Rev. 2004;25(3):389-425.

53. Giri M, Kaufman JM. Opioidergic modulation of in vitro pulsatile gonadotropin-releasing hormone release from the isolated medial basal hypothalamus of the male guinea pig. Endocrinology. 1994;135(5) 2137-2143.
54. Fraser LA, Morrison D, Morley-Forster P, et al. Oral opioids for chronic non-cancer pain: higher prevalence of hypogonadism in men than in women. Exp Clin Endocrinol Diabetes. 2009;117(1):38-43.

55. Fortin JD, Bailey GM, Vilensky JA. Does opioid use for pain management warrant routine bone mass density screening in men? Pain Physician. 2008;11(4):539-541.

56. De Maddalena C, Bellini M, Berra M, Meriggiola MC, Aloisi AM. Opioid-induced hypogonadism: why and how to treat it. Pain Physician. 2012;15(3 Suppl):SE111-SE118.

57. Brennan MJ. The effect of opioid therapy on endocrine function. Am J Med. 2013;126(3 Suppl 1):S12-S18.

58. Ceccarelli I, De Padova AM, Fiorenzani P, Massafra C, Aloisi AM. Single opioid administration modifies gonadal steroids in both the CNS and plasma of male rats. Neuroscience. 2006;140(3):929-937.

59. Aloisi AM, Aurilio C, Bachiocco V, et al. Endocrine consequences of opioid therapy. Psychoneuroendocrinology. 2009;34 Suppl 1: S162-S168.

60. Aurilio C, Ceccarelli I, Pota V, et al. Endocrine and behavioural effects of transdermal buprenorphine in pain-suffering women of different reproductive ages. Endocr J. 2011;58(12):1071-1078.

61. Pergolizzi J, Goehler K, Steigerwald I, Etopolski M, Kim M, Vorsanger G. Effects of Tapentadol on level of sexual hormones associated with opioid-induced androgen deficiency. Poster presented at: PainWeek 2012; Sept 5-8; 2012; Las Vegas, NV.

62. Mattia C. Editorial: non-analgesic effects of opioids: the dark side of the moon. Curr Pharm Des. 2012;18(37):5991-5993.
Therapeutics and Clinical Risk Management

\section{Publish your work in this journal}

Therapeutics and Clinical Risk Management is an international, peerreviewed journal of clinical therapeutics and risk management, focusing on concise rapid reporting of clinical studies in all therapeutic areas, outcomes, safety, and programs for the effective, safe, and sustained use of medicines. This journal is indexed on PubMed Central, CAS,

\section{Dovepress}

EMBase, Scopus and the Elsevier Bibliographic databases. The manuscript management system is completely online and includes a very quick and fair peer-review system, which is all easy to use. Visit http://www.dovepress.com/testimonials.php to read real quotes from published authors.

Submit your manuscript here: http://www.dovepress.com/therapeutics-and-clinical-risk-management-journal 